\title{
ON THE KINEMATICS OF THE LOCAL COSMIC VOID
}

\author{
O. G. Nasonova and I. D. Karachentsev \\ Special Astrophysical Observatory of the Russian Academy of Sciences, \\ Nizhnij Arkhyz, KChR, 369167, Russia
}

We collected the existing data on the distances and radial velocities of galaxies around the Local Void in the Aquila/Hercules to examine the peculiar velocity field induced by its underdensity. A sample of 1056 galaxies with distances measured from the Tip of the Red Giant Branch, the Cepheid luminosity, the SNIa luminosity, the surface brightness fluctuation method, and the Tully-Fisher relation has been used for this purpose. The amplitude of outflow is found to be $\sim 300 \mathrm{~km} \mathrm{~s}^{-1}$. The galaxies located within the void produce the mean intra-void number density about 1/5 of the mean external number density of galaxies. The void's population has a lower luminosity and a later morphological type with the medians: $M_{B}=-15.7^{m}$ and $T=8(\mathrm{Sdm})$, respectively.

Keywords: Galaxies - large-scale structure of the Universe.

\section{Introduction}

Since the cosmic voids were first detected (Joveer et al. 1978, Gregory \& Tompson 1978, Kirshner et al. 1981, de Laparent et al. 1986), it gradually became clear that numerous empty volumes with dimensions of $\sim 10-50 \mathrm{Mpc}$ are the main architectural elements of the large-scale structure of the Universe (van de Weygaert \& Platen, 2009). On the example of the Local Volume $(D<10 \mathrm{Mpc})$ it was shown (Tikhonov \& Karachentsev 2006) that there also exist smaller mini-voids and bubbles with a diameter of $\sim 1-5 \mathrm{Mpc}$. The closest of the existing cosmic lacunae was detected by Tully \& Fisher (1987) while compiling the Atlas of nearby galaxies. Its central part is located in the constellations of Aquila and Hercules, in the region of strong Galactic absorption. Nevertheless, an apparent deficit of galaxies in this region is only partially due to the absorption of light. The surveys of the Local Void in the $21 \mathrm{~cm}$ neutral hydrogen line, performed in the Parkes and Arecibo observatories (Zwaan et al. 2005, Kraan-Korteweg et al. 2008, Giovanelli et al. 2005, Saintonge et al. 2008) have confirmed low local density of galaxies with radial velocities below $3000 \mathrm{~km} \mathrm{~s}^{-1}$. Identifications of the IRAS sources accompanied by measurements of their radial velocities (Roman et al. 1996, Nakanishi et al. 1997), as well as the search for dwarf galaxies of low surface brightness (Karachentseva et al. 1998) did not eliminate the observed underrun of nearby galaxies in the region of the Local Void, which occupies about $1 / 6$ of the entire sky.

The dimensions of the Local Void in depth and the extent of its galaxy population are still debated. Tully et al. (2008) note that the region of low density extends up to distances of $\sim(40-60)$ Mpc. Kraan-Korteweg et al. (2008) suggest that the local region of depression may be even larger, neighboring a more distant void in Microscopium/Sagittarius. 
Furthermore, these authors denote the existence of a few filaments inside this volume, dividing the "supervoid" into 2 or 3 voids with the size of 10-30 Mpc.

This whole extended region with a low density of galaxies is located approximately along the north pole of the supergalactic coordinate system, that intensifies the concentration of nearby galaxies towards the Local Supercluster plane. According to results of numerical simulations in the $\Lambda$ CDM models (Ceccarelli et al. 2008, Schaap 2007, Tully et al. 2008), the expansion of cosmic voids occurs more rapidly and is characterized by a local excess of the Hubble constant $\Delta H \simeq 0.2 H_{0}$. At the typical void radius of $\sim 15 \mathrm{Mpc}$, peculiar velocities of galaxies revealed at its edges amount to around $\pm(220) \mathrm{km} \mathrm{s}^{-1}$. As Tully et al. (2008) argue, the presence of a vast Local Void in the direction of +SGZ generates the velocity of $\left(V_{p e c}\right)_{L G} \simeq 260 \mathrm{~km} \mathrm{~s}^{-1}$ of the Local Group relative to the cosmic microwave background. The direction of this component towards -SGZ explains a wellknown phenomenon of the "Local Velocity Anomaly" (Faber \& Burstein 1988), which remained a mystery during the past 20 years.

The observational data (Karachentseva et al. 1999, Kraan-Korteweg et al. 2008) show that the Aquila/Hercules region with the coordinates $\mathrm{RA}=\left[17.0^{h}-21.0^{h}\right]$ and Dec $=\left[-30^{\circ},+40^{\circ}\right]$ reveals an almost complete lack of galaxies with radial velocities below $1500 \mathrm{~km} \mathrm{~s}^{-1}$. This region of the sky is demonstrated in Fig. 1, where the shaggy diagonal band marks the region of strong absorption $A_{B}>2.0^{m}$ according to Schlegel et al. (1998). The galaxies with radial velocities $V_{L G}<1500 \mathrm{~km} \mathrm{~s}^{-1}$ in the Local Group rest frame are marked with circles, while the figures indicate the radial velocity of a given galaxy. In the velocity interval of [1600-2200] $\mathrm{km} \mathrm{s}^{-1}$ in this region there appear the galaxies that are likely to belong to the far wall of the nearby void, or to the filament that splits a larger void into sectors.

Judging from the distribution of galaxies in Fig. 1, the geometric center of the Local Void is located near $\mathrm{RA}=19.0^{h}$, Dec $=+3^{\circ}$, which practically coincides with the position of its center according to Tikhonov \& Karachentsev (2006). Note that other estimates appeared in the literature: $\mathrm{RA}=18.8^{h}, \mathrm{Dec}=-3^{\circ}$ (Tully et al. 2008), and $\mathrm{RA}=18.6^{h}$, $\mathrm{Dec}=+18^{\circ}$ (Karachentseva et al. 1999).

Below we consider the structure and kinematics of the Local Void as the near part of a more extended depression among the galaxies observed along the north pole of the Local Supercluster. To be definite, we assume that the Local Void begins right at the edge of the Local Group and extends approximately $20 \mathrm{Mpc}$, with the center at RA=19.0 $0^{h}$, $\mathrm{Dec}=+3^{\circ}$ at the distance of $D_{L V}=10.0 \mathrm{Mpc}$ from the observer.

\section{Observational data}

In order to analyze the peculiar velocity field in the vicinity of the Local Void, we collected a sample of galaxies with known radial velocities and distances that are located within a spherical volume of radius $R_{L V}=25 \mathrm{Mpc}$ around the void center fixed above. Such a sufficiently large radius, 2.5 times the assumed radius of the void, was selected to have a 


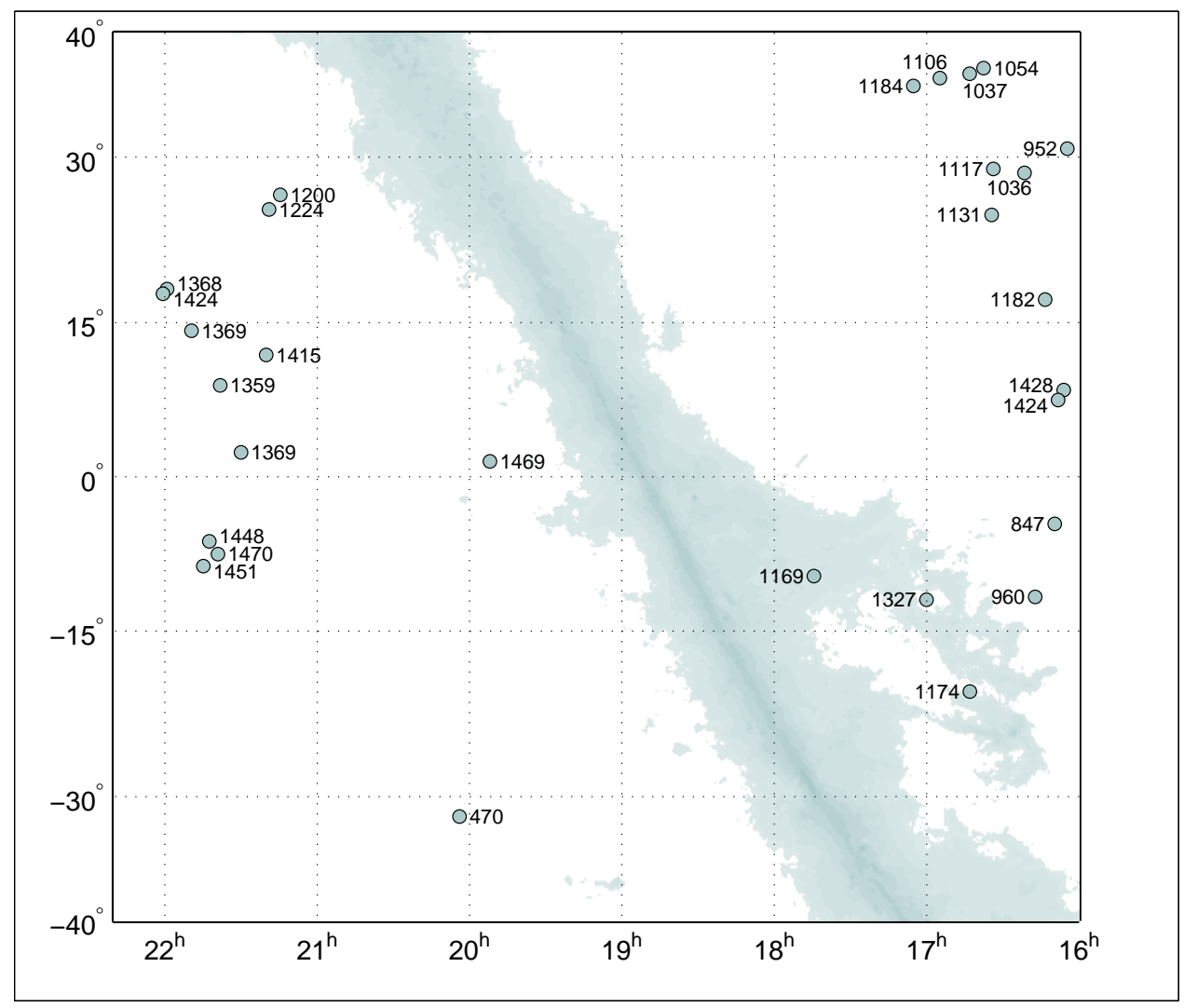

Fig. 1. Distribution of galaxies in the sky in the Local Void region in equatorial coordinates. The numbers indicate the radial velocity of galaxies relative to the Local Group centroid. The diffuse band depicts the zone of strong galactic absorption.

representative asymptotics of the velocity field around the void. The NED and HyperLeda databases were our sources of data on the radial velocities of galaxies. Radial velocities were expressed relative to the centroid of the Local Group, with the apex parameters used in the NED.

The distances to the galaxies in our sample were measured in different ways. For nearby galaxies, the most versatile and accurate method of distance estimation is to use the luminosity of the Tip of the Red Giant Branch (TRGB). The application of this method, proposed by Lee et al. (1993), to the images of galaxies, obtained with the WFPC2 and ACS cameras of the HST, gave accurate distances to more than 250 galaxies in the Local Volume. A summary of TRGB distances is contained in the Catalog of Neighboring Galaxies (=CNG,Karachentsev et al. 2004). Using the CNG, we have adjoined more recent estimates of distances (Karachentsev et al. 2006, Tully et al. 2006), as well adding to them a number of galaxies with distances measured from the luminosity of Cepheids.

For the early type $(\mathrm{E}, \mathrm{S} 0)$ galaxies dominated by old stellar population, the most effective method to measure distances is based on the fluctuations of surface brightness. Tonry et al. (2001) measured by this method the distances to $300 \mathrm{E}$ and S0 galaxies with 
Table 1: Number of galaxies around the Local Void with distances measured by different methods.

\begin{tabular}{lrcr}
\hline Sample & Number & $\sigma_{m}$ & Goodness \\
\hline TRGB+Ceph & 264 & 0.15 & 10.8 \\
SBF (Tonry) & 141 & 0.25 & 4.7 \\
SNIa (Tonry) & 11 & 0.10 & 3.3 \\
TF (NIR) & 139 & 0.40 & 2.9 \\
TF (opt) & 501 & 0.40 & 5.6 \\
\hline All & 1056 & - & 27.3 \\
\hline
\end{tabular}

typical velocities $c z<4000 \mathrm{~km} \mathrm{~s}^{-1}$.

We have as well included in our sample a small number of galaxies with high-precision distance measurements from the luminosity of SNIa type Supernovae (Tonry et al. 2003).

Kashibadze (2008) used a multiparametric near-infrared Tully-Fisher relation to determine distances to $\sim 400$ spiral galaxies from the 2MASS Selected Flat Galaxy Catalog by Mitronova et al. (2004) with radial velocities $c z<3000 \mathrm{~km} \mathrm{~s}^{-1}$. The zero-point of this NIR-TF relation was calibrated by 15 galaxies with distance estimated from the Cepheids and TRGB.

Finally, we used compilations of distance estimates based on the classic optical (oneparametric) Tully-Fisher relations in different photometric bands (B, R, I), presented by Tully et al. (2008, 2009) and Springob et al. (2007). To calibrate these data we used the galaxies with distances measured via the TRGB and Cepheids.

In total our sample contains 1056 galaxies. The distribution of their number by the method of distance estimation is presented in Table 1. The last two columns of the Table indicate the distance modulus measurement error, typical for each method, and a statistical significance of each subsample i.e. its "goodness", $G=(N / 100)^{1 / 2} \cdot \sigma_{m}^{-1}$.

Figure 2 shows the distribution in the sky of 1056 galaxies from our sample in equatorial coordinates. Galaxies with close $(R<10 \mathrm{Mpc})$, medium (10-20 Mpc) and far $(R>20 \mathrm{Mpc})$ distances are shown by circles of different diameters. The region of strong absorption $\left(A_{B}>2.0^{m}\right)$ is filled with gray. The members of the Local Group with $R<1.1 \mathrm{Mpc}$ were excluded. The Hubble relation between radial velocities and distances of 1056 galaxies relative to the observer is presented in Figure 3. The galaxies with distance estimates made using different methods are depicted by different symbols. The near part of the Hubble diagram $\left(R_{L G}<10 \mathrm{Mpc}\right)$ is dominated by the galaxies with highaccuracy distance measurements using the TRGB and Cepheids, and at larger distances their estimates are derived mainly from the Tully-Fisher diagrams, and the fluctuations of surface brightness. An increased scatter of galaxies relative to the line with an inclination of $H_{0}=73\left(\mathrm{~km} \mathrm{~s}^{-1}\right) / \mathrm{Mpc}$ (the dashed line) at $R_{L G} \simeq 15-20 \mathrm{Mpc}$ exists owing to the virial velocities of members of the Virgo cluster. 


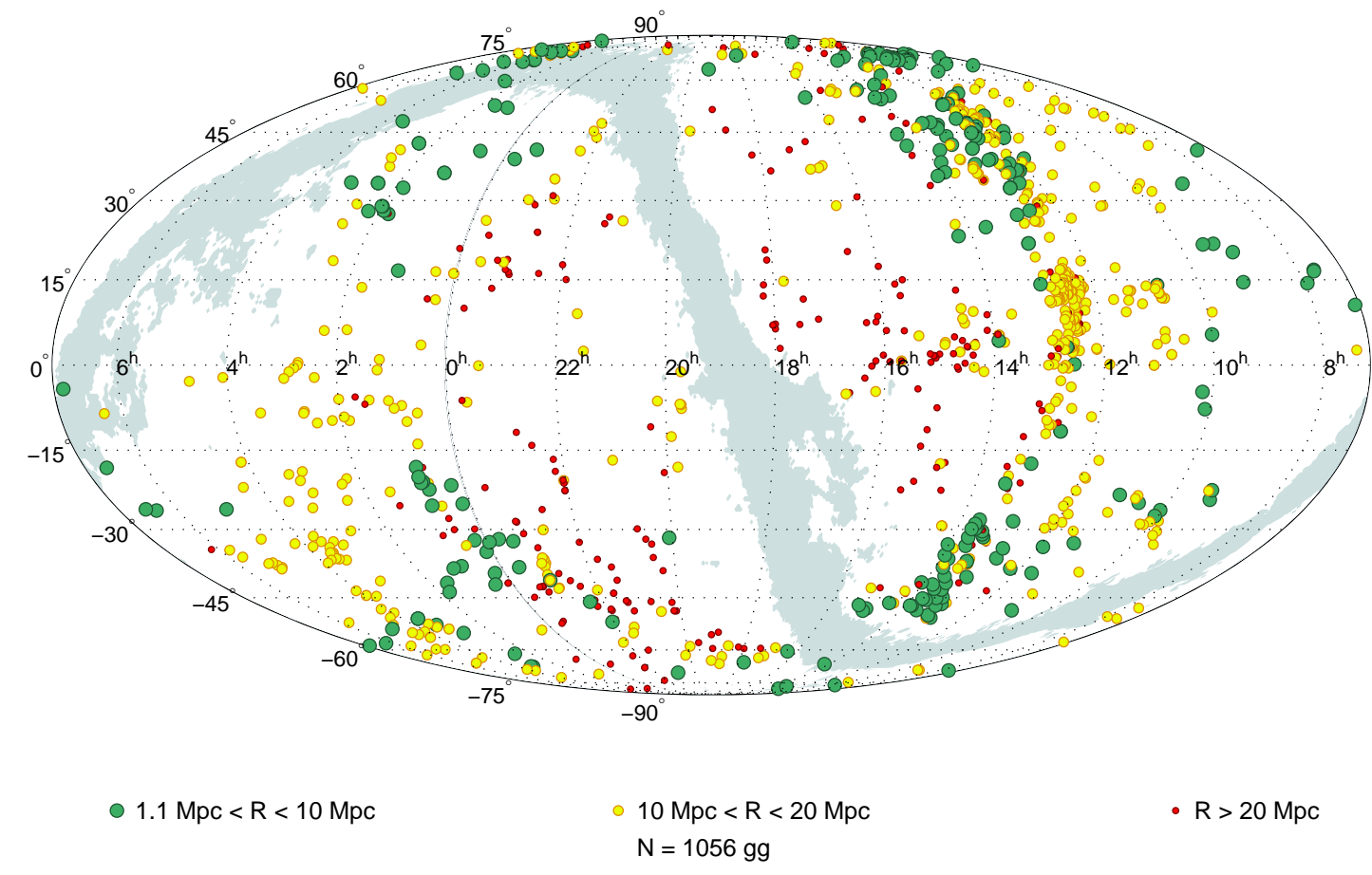

Fig. 2. Distribution of 1056 galaxies in the sky with distances from the center of the Local Void within $25 \mathrm{Mpc}$. The galaxies in three intervals of distance from the observer are marked with circles of different diameters.

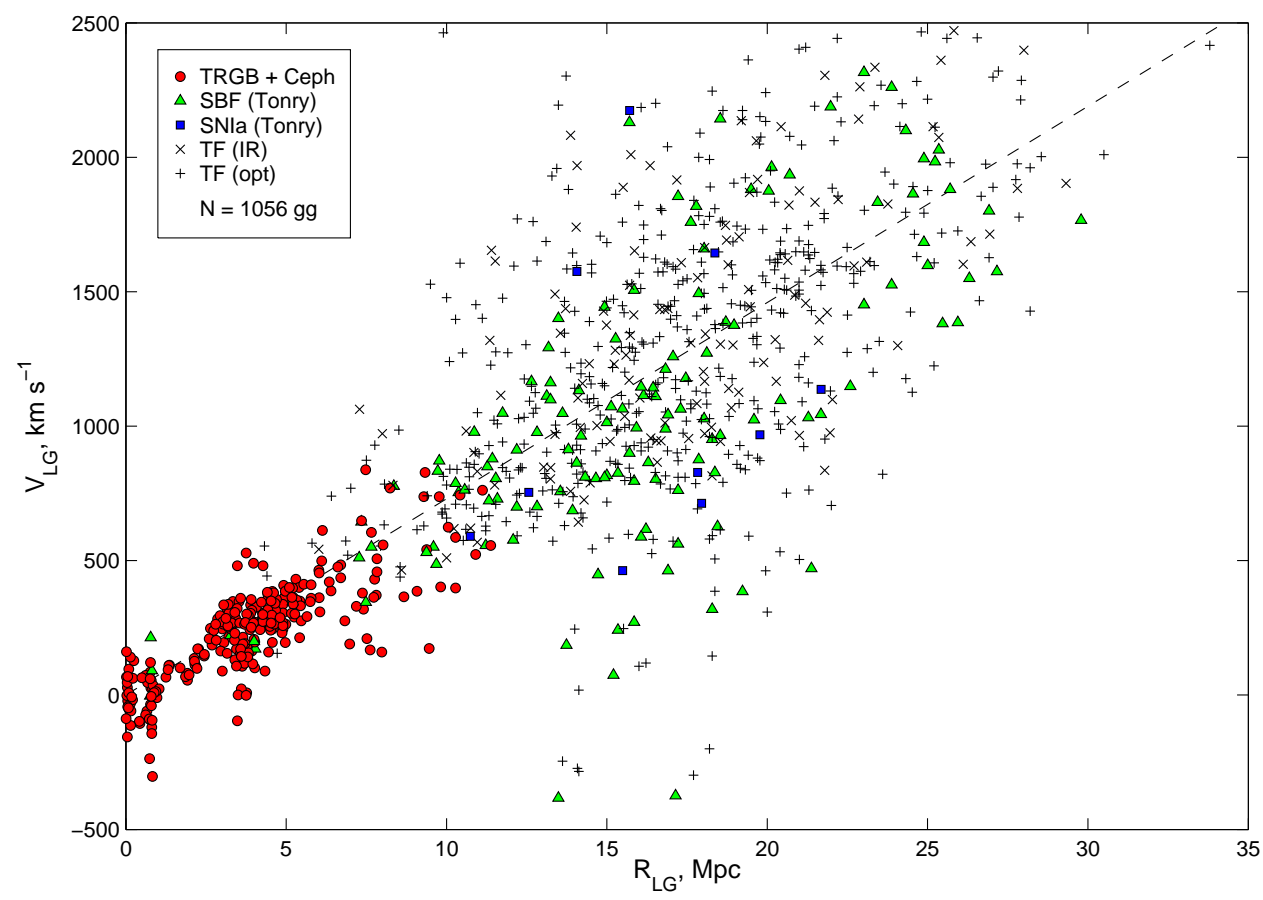

Fig. 3. Distribution of 1056 galaxies by radial velocities and distances from the observer. Galaxies with different sources of distances are marked with different symbols. 


\section{Discussion}

When there exists an expansion of the void's neighborhood, then the galaxies, located for the observer in front of the empty volume, should have the velocities systematically lower than the Hubble velocity, and the objects behind the far edge of the void shall have radial velocities higher than the Hubble velocity. The pattern of deviations from the pure Hubble flow will be characterized by a wave with an amplitude of an opposite sign to that, observed in the pattern of the Virgo-centric flow and Fornax-centric flow around these nearby attractors (Karachentsev \& Nasonova 2010, Nasonova et al. 2011). Here the amplitude of outflow velocity from the void is expected to be the lower, the further the angular distance of a galaxy $\Theta$ from the the void center.

The top and bottom panels in Fig. 4 reproduce the Hubble diagrams for 24 and 104 galaxies, located in the cones of $\Theta<30^{\circ}$ and $30^{\circ}<\Theta<60^{\circ}$, respectively. Since the Local Void begins literally at the doorstep of the Local Group, we can not actually detect the nearby part of the "outflow wave". Some of the closest galaxies at $R_{L G} \simeq 1 \mathrm{Mpc}$ are the members of the Local Group. On the far side behind the void, in the $\Theta<30^{\circ}$ cone the anticipated excess of radial velocities of galaxies is observed. The broken line in the figure represents a sliding median with the averaging window of $2 \mathrm{Mpc}$. Its excess over the Hubble line at $H_{0}=73\left(\mathrm{~km} \mathrm{~s}^{-1}\right) / \mathrm{Mpc}$ reaches $\sim 500 \mathrm{~km} \mathrm{~s}^{-1}$. Unfortunately, all but one of the galaxies in this diagram have a low accuracy of distance measurements.

At the bottom plot of Fig. 4 the effect of galaxy outflows from the Local Void is noticeable only as a trend. The median radial velocity follows on the average by $10-15 \%$ higher than the global value of $H_{0}=73\left(\mathrm{kms}^{-1}\right) / \mathrm{Mpc}$, which is consistent with theoretical expectations. One galaxy with a high-precision distance of 7.8 $\pm 0.6 \mathrm{Mpc}$ (KK 246=ESO 461-36) is located within the void at its eastern near side and has a negative peculiar velocity to us of about $110 \mathrm{~km} \mathrm{~s}^{-1}$.

It must be emphasized that the Hubble diagrams in Fig. 4 do not take into account the complete observational data on the kinematics of galaxies, surrounding the void. For this reason, we used also another approach: building a Hubble diagram, but for velocities and distances of galaxies relative to the center of the Local Void. At the distance of a galaxy from the observer $R_{g}$, and the distance of the void's center from the observer $R_{c}$, the squared distance of the galaxy from the void center is

$$
R_{L V}^{2}=R_{g}^{2}+R_{c}^{2}-2 R_{g} R_{c} \cos \Theta
$$

However, in contrast to the distance, a transition from the radial velocity of the galaxy to its velocity relative to the void center is ambiguous as we do not know the complete velocity vector of the galaxy, namely, its tangential component. We viewed this issue on an example of the Virgo-centric flow (Karachentsev et al. 2010). In the case when

galaxies are involved in a weakly perturbed Hubble flow (a model of a small void), their velocity relative to the center of the void is expressed as

$$
V_{L V}=V_{g} \cos \lambda-V_{c} \cos (\lambda+\Theta),
$$



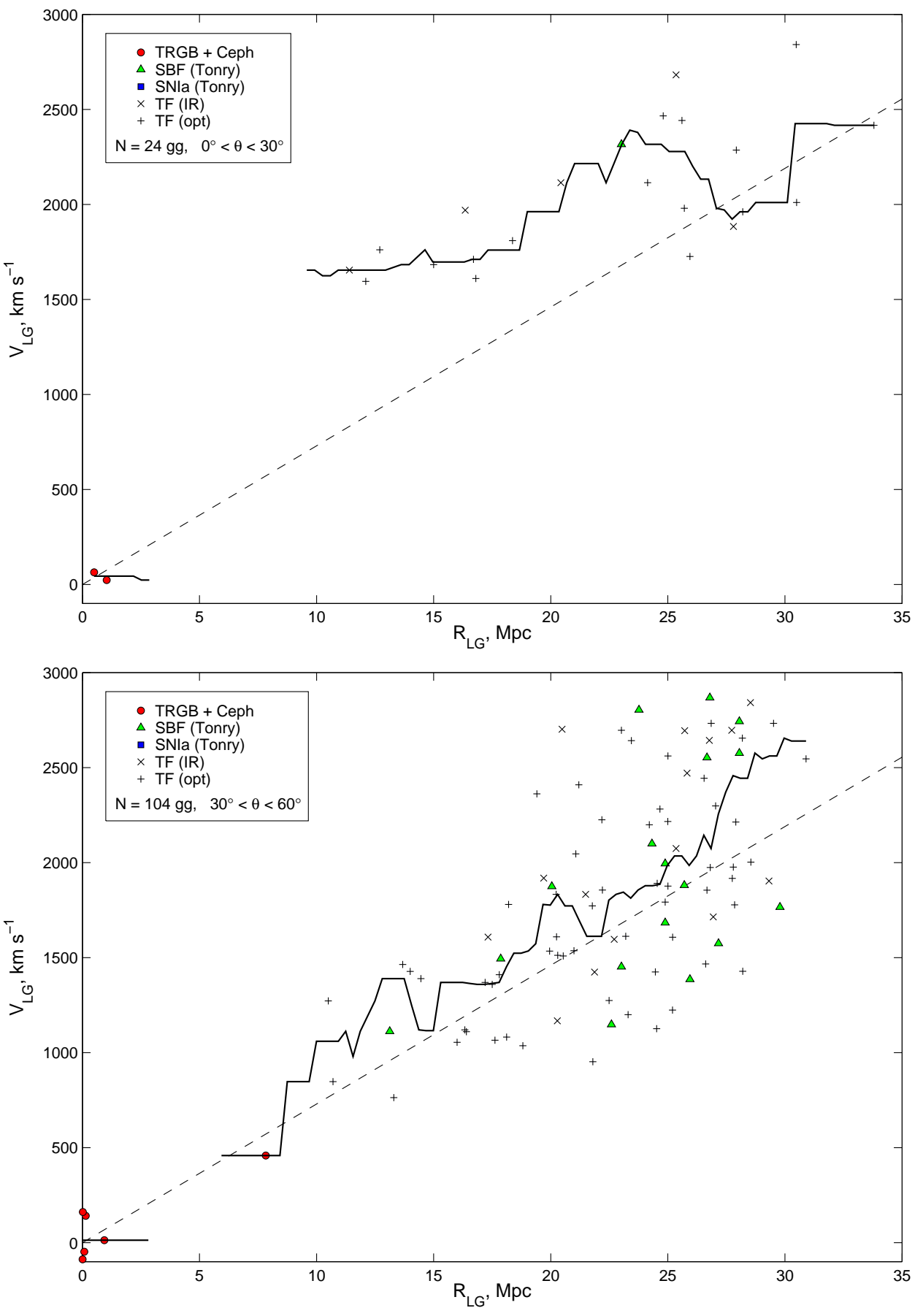

Fig. 4. Hubble diagram for galaxies with angular distances from the Local Void center below $30^{\circ}$ (top panel) and $30-60^{\circ}$ (bottom panel). 
where $V_{g}$ and $V_{c}$ denote the radial velocities of the galaxy and the void center, and $\lambda$ is the angle between the line of sight and the line connecting the galaxy with the void center:

$$
\tan \lambda=R_{c} \sin \Theta /\left(R_{g}-R_{c} \cos \Theta\right) .
$$

In another extreme case, when the dominant motion of galaxies is their radial recession from the void (a model of extended void), the velocity of galaxies relative to the void center is expressed as

$$
V_{L V}=\left(V_{g}-V_{c} \cos \Theta\right) \sec \lambda
$$

(see Fig. 2 in Karachentsev et al. 2010). The differences between the two assumed schemes of galaxy motions become significant at the angles $45^{\circ}<\lambda<135^{\circ}$.

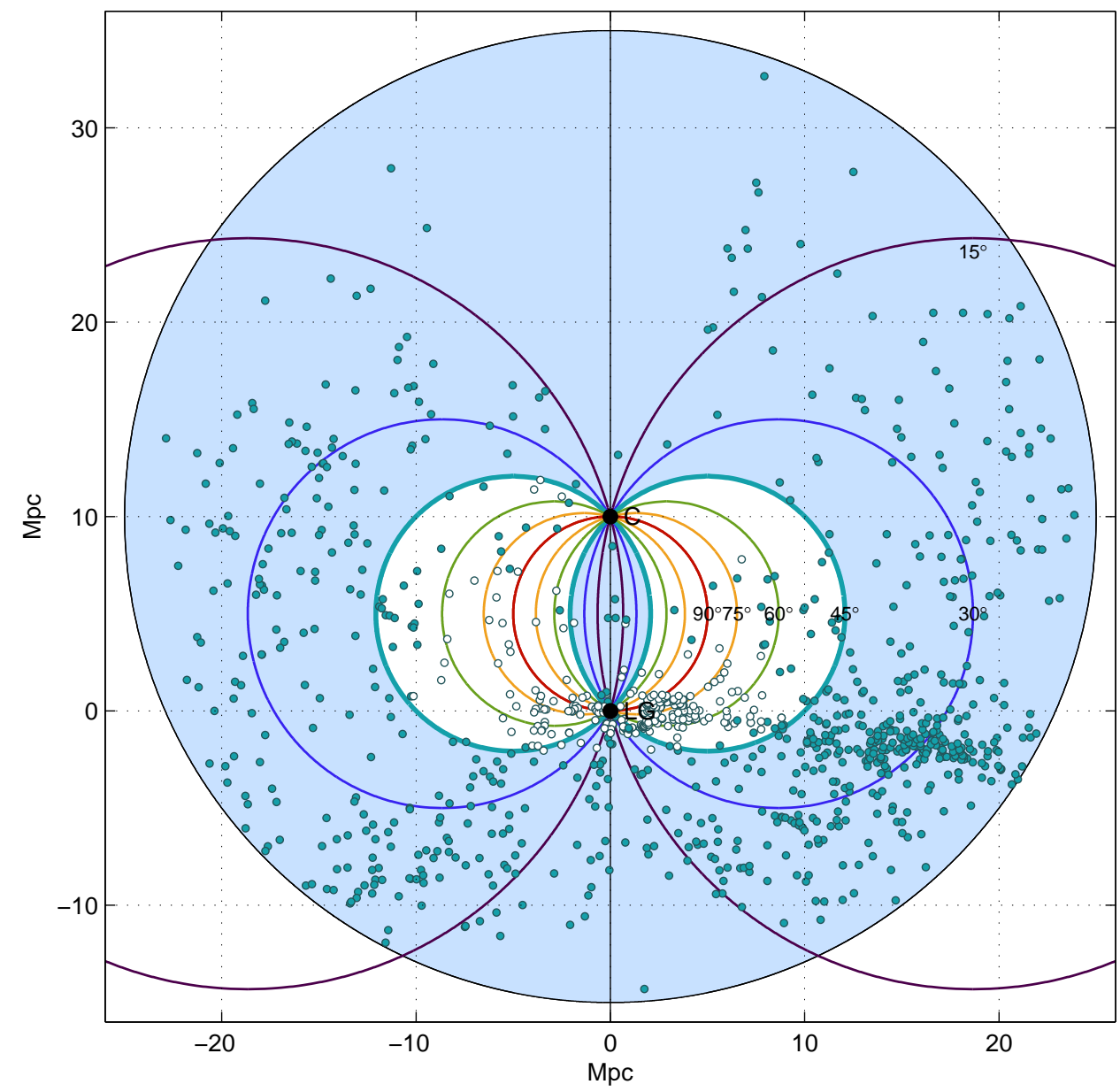

Fig. 5. Distribution of galaxies around the Local Void projected on the plane passing through the Local Group (LG) and the Void center (C). The circles correspond to the lines on which the angle $\lambda$ between the line of sight and the direction from the galaxy towards the center of the void takes the values: $15^{\circ}, 30^{\circ}, \ldots 90^{\circ}$. The galaxies, located in the zone of "unfavorable "values of $45^{\circ}<\lambda<135^{\circ}$ are shown with empty circles. 
Figure 5 illustrates the situation, showing the distribution of 1056 galaxies with known velocities and distances within a radius of $R_{V C}=25 \mathrm{Mpc}$ around the center of the Local Void in Cartesian coordinates. The distribution of these galaxies is projected onto the plane passing through the Local Group (LG) and the Local Void center (C). The selected projection plane approximately corresponds to the Supergalactic plane \{SGZ, SGY\}, where the concentration of galaxies in the lower right part exists owing to the Virgo cluster. The lines of the fixed angle $\lambda=15^{\circ}, 30^{\circ}, \ldots 90^{\circ}$ are marked in the Figure with symmetrical circles. The galaxies with angles $45^{\circ}<\lambda<135^{\circ}$ are characterized by the greatest uncertainty of transition from $V_{g}$ to $V_{L V}$ due to an unknown tangential component of their velocity relative to the observer. These "bad" galaxies are shown with open circles. In space the region $45^{\circ}<\lambda<135^{\circ}$ has the form of a torus, the projection of which in Fig. 5 looks like a white apple.

The distribution of galaxies by velocity and distance from the center of the Local Void is presented in Fig. 6. The upper panel represents the distribution of all the 1056 galaxies, while the bottom plot shows the $\left\{V_{L V}, R_{L V}\right\}$ distribution only for 777 galaxies with corresponding angles of $\lambda<45^{\circ}$ or $\lambda>135^{\circ}$. The dashed line in the panels corresponds to the unperturbed Hubble flow with the parameter $H_{0}=73\left(\mathrm{~km} \mathrm{~s}^{-1}\right) / \mathrm{Mpc}$, and the broken solid line represents the sliding median with a window of $2 \mathrm{Mpc}$. In both panels outside $R_{L V}=10 \mathrm{Mpc}$, the sliding median reasonably follows the linear Hubble relation, and at $R_{L V}<10 \mathrm{Mpc}$ it reveals a velocity excess of $\sim 300 \mathrm{~km} \mathrm{~s}^{-1}$, which clearly indicates an accelerated expansion of the void's edges. While constructing these diagrams the observed radial velocities of galaxies were converted into the velocities $V_{L V}$ based on the model of a small void. The use of another assumption (the model of extended void) yields roughly the same picture for 777 galaxies with angles $\lambda<45^{\circ}$ or $\lambda>135^{\circ}$.

\section{Galaxies within the Local Void}

Current ideas on the structure of empty cosmic volumes admit the existence inside of them of a small number of galaxies, generally of low luminosity (Peebles 2001, Patiri et al. 2006, Hoeft \& Gottloeber 2010). An observational verification of this hypothesis turns out to be rather difficult, and can be successful only for the closest voids. Distribution of the considered galaxies in the vicinity of the Local Void is presented in the plots in Fig. 7 being projected in the Supergalactic $\{$ SGZ, SGY $\}$ and $\{$ SGZ, SGX $\}$ planes. To highlight the contours of the Void, the plots contain the galaxies within $\pm 5 \mathrm{Mpc}$ layers from the respective planes. The contrast of the Local Void is notable here, however, it is expressed most distinctly if the line of sight is turned by the angle $\sim 45^{\circ}$ relative to SGX.

With the distance estimates from the Local Void center for all the galaxies, we determined the variation in the number density of galaxies along the radius of the Void. We used their mean number density in the sphere of radius $R_{L V}=25 \mathrm{Mpc}$ as normalization. The resulting profile of the Local Void is presented in Fig. 8. Here the solid circles outline the density profile with the position of the Void center at the point $\left\{\mathrm{RA}=19.0^{h}, \mathrm{Dec}=+3^{\circ}\right\}$, 

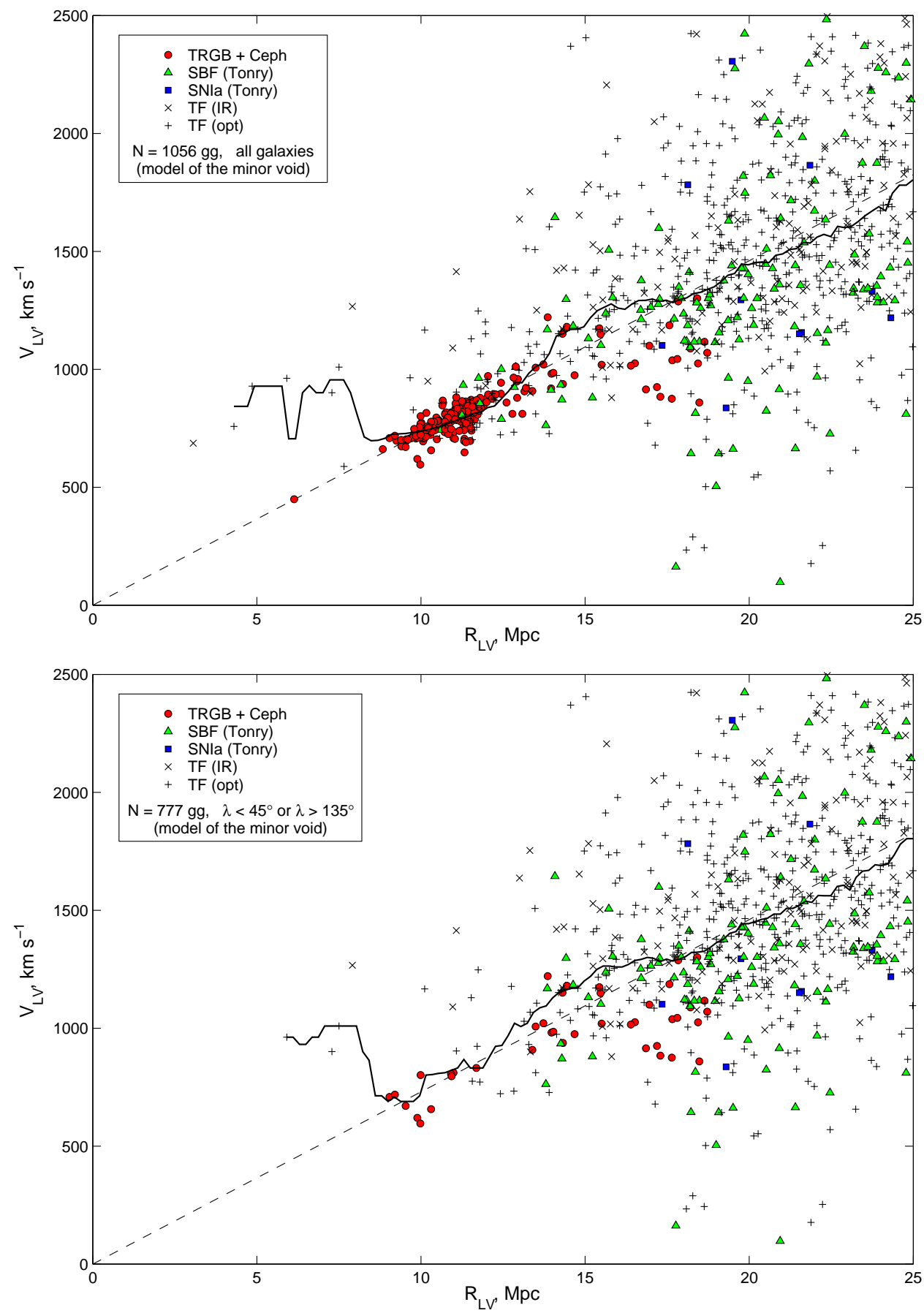

Fig. 6. The relationship between the velocities and distances of galaxies relative to the center of the Local Void. The subsamples of galaxies with distances estimated via different methods are marked with the same symbols as in Fig.3. The top plot represents all the galaxies, the bottom plot - the galaxies with a "favorable" orientation angles $\lambda$. 

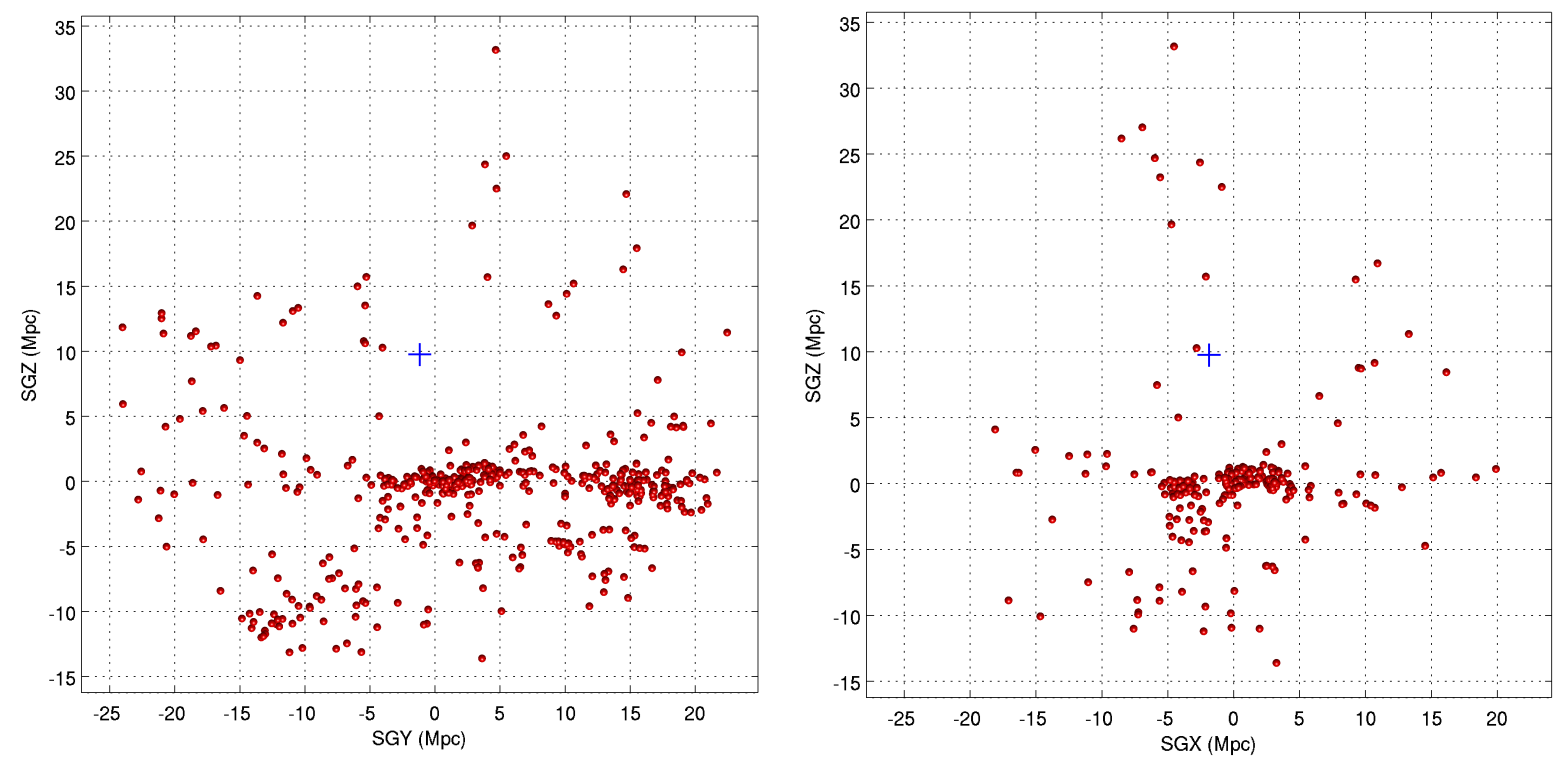

Fig. 7. Distribution of galaxies around the Local Void in projection on the Supergalactic $\{$ SGZ, SGY $\}$ and $\{$ SGZ, SGX $\}$ planes. The position of the void's center is indicated by the cross. The left and right panels demonstrate only the galaxies within the layer $|\mathrm{SGX}|<5 \mathrm{Mpc}$ and $|\mathrm{SGY}|<5 \mathrm{Mpc}$, respectively.

and the empty circles correspond to the center position at $\left\{\mathrm{RA}=18.6^{h}, \mathrm{Dec}=+18^{\circ}\right\}$. As we see, general shape of the $n\left(R_{L V}\right) / \bar{n}$ profile changes little when the void center is shifted. Inside the void, the mean number density of galaxies amounts to about $1 / 5$ of the average density. Directly at the edge of the void at $R_{L V} \simeq(10-12)$ Mpc there is a peak density, which is obviously formed by the filaments imbordering the void. It should be noted that the nearby half of the Local Void is practically free from galaxies down to the absolute magnitude $M_{B} \simeq-10^{m}$, whereas the far half of the void reveals galaxies, the distances to which are measured with $\mathrm{a} \sim 20 \%$ error, i.e. $\sim(3-4)$ Mpc. Therefore, the reality of galaxies being present within the void needs further confirmations with more accurate measurements of their distances.

Some of the data on 16 galaxies within the Local Void are presented in Table 2. In addition to the distances and velocities relative to the Local Group, the columns contain: the distances from the void center $R_{L V}$ (in $\mathrm{Mpc}$ ), velocities relative to the center of the void (in $\mathrm{km} \mathrm{s}^{-1}$ ) when calculated according to the model of a small and extended void, as well as the morphological types and absolute magnitudes of the galaxies. The galaxies are ranked by the distance value $R_{L V}$. The column " $\lambda$ " lists the values of the angle, characterizing the galaxy position relative to the line of sight and the direction towards the center of the void.

It follows from the data above that the sparse population of the Local Void mostly contains the galaxies of late types and low luminosity. The median absolute magnitude of galaxies in Table 2 is $-15.7^{m}$, and the median morphological type corresponds to $T=8$ or Sdm. The only early-type galaxy in the Table, KKR 25, belongs to the rare type of 


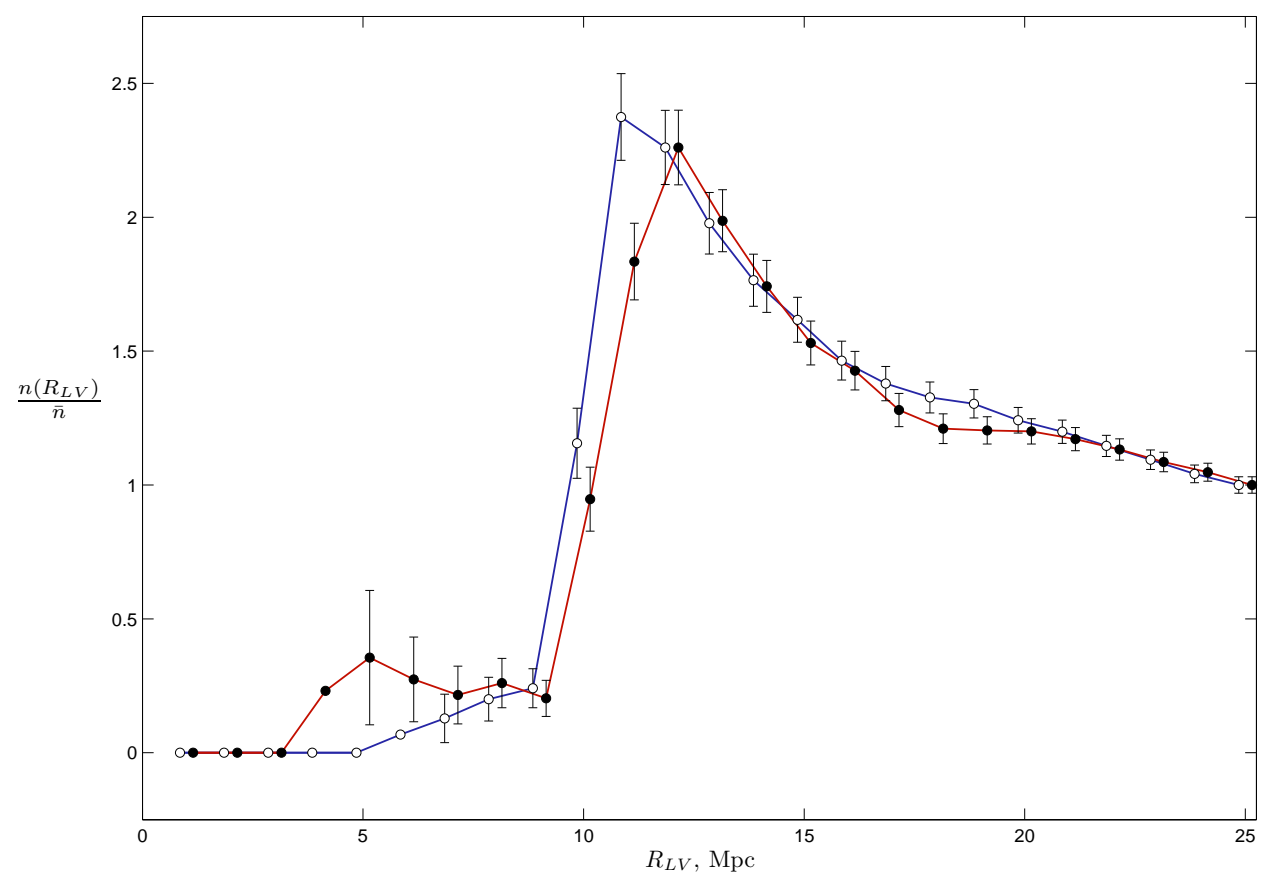

Fig. 8. Density number profile of galaxies along the radius of the Local Void at two positions of its center.

isolated dwarf spheroidal galaxies. Due to their very low luminosity $\left(M_{B}=-9.9^{m}\right)$, such objects are difficult to detect, but can be quite common in the space between groups and clusters (Karachentsev et al. 2009).

\section{Concluding remarks}

We did not find any detailed data in the literature on the structure and kinematics of a typical cosmic void. Clearly, the numerical simulations of the process of void's expansion under the $\Lambda \mathrm{CDM}$ paradigm allow one to draw in Figures 4 and 6 the regression lines, which give a quantitative description of the expansion pattern. According to Tully et al. (2008), the expansion of absolutely empty spherically symmetric void at $\Omega_{\lambda}=0.76$ is characterized by the local Hubble constant $H_{v} \simeq H_{0}+16\left(\mathrm{~km} \mathrm{~s}^{-1}\right) / \mathrm{Mpc}$. Then, at the diameter of the Local Void we adopted as $20 \mathrm{Mpc}$, the amplitude of outflow velocity differential amounts to $\sim 320 \mathrm{~km} \mathrm{~s}^{-1}$. This value is quite consistent with the available observational data. Of course, the kinematics of a real nonspherical void may differ significantly from this simplest model.

It should be noted that we have not yet exhausted the observational capabilities to clarify the expansion pattern of the Local Void. In its near side there are some galaxies, the distances to which can be measured using the TRGB method with an accuracy better than $5 \%$. At the far edge of the void, there remain a lot of spiral galaxies that are seen nearly edge-on, and for which so far there are no accurate measurements of the HI line 
Table 2: Galaxies nearest to the Local Void center $\left(\mathrm{RA}=19.0^{h}, \mathrm{Dec}=+3^{\circ}\right)$

\begin{tabular}{lcrrrrrrr}
\hline Galaxy & $\mathrm{RA}(2000.0) \mathrm{Dec}$ & $R_{L G} \pm \sigma$ & $V_{L G}$ & $R_{L V}$ & $V_{L V}$ & $\mathrm{~T}$ & $M_{B}$ & $\lambda$ \\
\hline 2MFGC15085 & $194311.7-065621$ & $11.4 \pm 2.3$ & 1654 & 3.06 & $687 / 1683$ & 8 & -17.2 & 56 \\
UGCA417 & $200921.2-061710$ & $12.1 \pm 2.4$ & 1595 & 4.30 & $758 / 1455$ & 8 & -15.5 & 51 \\
NGC6835 & $195433.1-123409$ & $12.7 \pm 2.5$ & 1761 & 4.86 & $928 / 1567$ & 6 & -18.1 & 47 \\
NGC6821 & $194424.1-064960$ & $15.0 \pm 3.0$ & 1683 & 5.91 & $962 / 1084$ & 8 & -17.1 & 26 \\
KK246 & $200357.4-314054$ & $7.8 \pm 0.6$ & 458 & 6.14 & $449 / 11257$ & 10 & -13.7 & 91 \\
CGCG371-004 & $194326.0-011032$ & $16.8 \pm 3.4$ & 1610 & 7.29 & $901 / 931$ & 5 & -18.7 & 16 \\
CGMW 3-4603 & $194238.0-073639$ & $16.7 \pm 3.3$ & 1711 & 7.50 & $1009 / 1072$ & 8 & -17.0 & 20 \\
MCG-01-41-6 & $160936.8-043713$ & $10.7 \pm 2.1$ & 847 & 7.65 & $588 / 706$ & 9 & -15.6 & 64 \\
2MFGC14044 & $174804.5+144429$ & $16.3 \pm 3.3$ & 1969 & 7.91 & $1267 / 1450$ & 8 & -17.7 & 27 \\
N6789 & $191641.8+635822$ & $3.6 \pm 0.3$ & 144 & 8.84 & $662 / 1491$ & 9 & -14.3 & 98 \\
SagDIG & $192959.0-174041$ & $1.0 \pm 0.1$ & 23 & 9.04 & $708 / 718$ & 10 & -11.5 & 156 \\
DDO210 & $204651.8-125053$ & $0.9 \pm 0.1$ & 13 & 9.21 & $718 / 739$ & 10 & -11.1 & 146 \\
KKR25 & $161347.6+542216$ & $1.9 \pm 0.2$ & 68 & 9.26 & $697 / 891$ & -1 & -9.9 & 108 \\
IC 4662 & $174706.3-643825$ & $2.4 \pm 0.2$ & 145 & 9.40 & $691 / 957$ & 9 & -15.6 & 97 \\
IC 5152 & $220241.9-511743$ & $2.0 \pm 0.2$ & 75 & 9.40 & $700 / 1012$ & 9 & -15.7 & 102 \\
N6503 & $174927.6+700841$ & $5.3 \pm 0.4$ & 301 & 9.41 & $673 / 177$ & 6 & -18.1 & 81 \\
\hline
\end{tabular}

profiles, and no reliable photometry. With the view of the current GBT monitoring such kind galaxies (Courtois et al. 2009), and the photometric PanStarr survey, new and more reliable observational data on the kinematics of the Local Void may appear in the near future.

\section{Acknowledgements}

Authors thank Brent Tully for useful discussions and valuable comments.

We made use of the HyperLEDA (http://leda.univ-lyon1.fr/) and NED (http://nedwww.ipac.caltech.edu/) databases.

This work was supported by the grants: RFBR 09-02-90414-Ukr-f-a and

RFBR 10-02-92650.

\section{References}

1. Ceccarelli L., Padilla N.D., Valotto C., Lambas D. G., 2008, arXiv:0805.0797

2. Courtois H., Tully B., Fisher R. et al. 2009, AJ, 138, 1938

3. de Lapparent V., Geller M. J., Huchra J. P., 1986, ApJ, 302, L1

4. Faber S. M., Burstein D., 1988, in "Large-Scale Motions in the Universe", A Vatican study Week, p.115

5. Giovanelli R., et al., 2005, AJ, 130, 2598

6. Gregory S. A., Thompson L. A., 1978, ApJ, 222, 784

7. Hoeft M., Gottloeber S., 2010, arXiv:1001.4721 
8. Joeveer M., Einasto J., Tago E., 1978, MNRAS, 185, 357

9. Karachentsev I. D., Nasonova (Kashibadze) O. G., 2010, MNRAS, 405, 1075

10. Karachentsev I.D., Kashibadze O.G., Makarov D.I., Tully R.B., 2009, MNRAS, 393, 1265

11. Karachentsev I.D., Dolphin A.E., Tully R.B., et al., 2006, AJ, 131, 1361

12. Karachentsev I.D., Karachentseva V.E., Huchtmeier W.K., Makarov D.I., 2004, AJ, 127, 2031 (= CNG)

13. Karachentseva V. E., Karachentsev I.D., Richter G. M., 1998, A\&AS, 134, 1

14. Kashibadze O. G., 2008, Astrofizika, 51, 409

15. Kraan-Korteweg R. C., Shafi N., Koribalski B.S., et al., 2008, in "Galaxies in the Local Volume", ASSP, p.13

16. Lee M. G., Freedman W. L. Madore B. F., 1993, ApJ, 417, 553

17. Mitronova S.N., Karachentsev I.D., Karachentseva V.E., Jarrett T.H., Kudrya Yu. N., 2004, BSAO, 57, 5 (2MFGC)

18. Nakanishi K., Takata T., Yamada T., et al., 1997, ApJS, 112, 245

19. Nasonova O.G., de Freitas Pacheco J.A., Karachentsev I.D., A\&A, submitted

20. Patiri S. G., Betancort-Rijo J., Prada F., et al., 2006, MNRAS, 372, 1710

21. Peebles P. J. E., 2001, ApJ, 557, 495

22. Roman A. T., Nakanishi K., Tomita A., Saito M., 1996, PASJ, 48, 679

23. Saintonge A., Giovanelli R., Haynes M.P., et al., 2008, AJ, 135, 588

24. Schaap W., 2007, PhD Thesis, Groningen Univ.

25. Schlegel D. J., Finkbeiner D. P. ES Davis M., 1998, ApJ, 500, 525

26. Springob C.M., Haynes M.P., Giovanelli R., Kent B. R., 2005, ApJS, 160, 149

27. Tikhonov A. V., Karachentsev I. D., 2006, ApJ, 653, 969

28. Tonry J.L., Schmidt B. P., Barris B., et al., 2003, ApJ, 594, 1

29. Tonry J.L., Dressler A., Blakeslee J.P., et al., 2001, ApJ, 546, 681

30. Tully R. B., Rizzi L., Shaya E. J., Courtois H. M., Makarov D.I., Jacobs B. A., 2009,

AJ, 138, 323

31. Tully R. B., Shaya E. J., Karachentsev I. D., et al., 2008, ApJ, 676, 184

32. Tully R. B., Rizzi L., Dolphin A. E., et al., 2006, AJ, 132, 729

33. Tully R.B., Fisher J.R., 1987, Nearby Galaxies Atlas, Cambridge Univ. Press, Cambridge

34. van de Weygaert R., van Kampen E., 1993, MNRAS, 263, 481

35. Zwaan M.A., Staveley-Smith L., Koribalski B.S., et al., 2003, AJ, 125, 2842 